\title{
(6) OPEN ACCESS \\ Clinical and molecular characterisation of hyperinsulinaemic hypoglycaemia in infants born small-for-gestational age
}

\author{
Ved Bhushan Arya, ${ }^{1}$ Sarah E Flanagan, ${ }^{2}$ Anitha Kumaran, ${ }^{1}$ Julian P Shield ${ }^{3}$ \\ Sian Ellard, ${ }^{2}$ Khalid Hussain, ${ }^{1}$ Ritika R Kapoor ${ }^{1}$
}

\begin{abstract}
${ }^{1}$ Developmental Endocrinology Research Group, Molecular Genetics Unit, London Centre for Paediatric Endocrinology and Metabolism, Great Ormond Street Hospital for Children NHS Trust, and The Institute of Child Health, University College London, London, UK

${ }^{2}$ Institute of Biomedical and Clinical Science, University of Exeter Medical School, Exeter, UK

${ }^{3}$ Department of Child Health, University of Bristol, and Bristol Royal Hospital for Children, Bristol, UK
\end{abstract}

\section{Correspondence to} Dr Ritika R Kapoor, Developmental Endocrinology Research Group, Molecular Genetics Unit, Institute of Child Health, University College London, 30 Guilford Street, London WC1N 1EH, UK; sejjrik@ucl.ac.uk

Received 23 August 2012 Revised 3 January 2012 Accepted 4 January 2013 Published Online First 29 January 2013

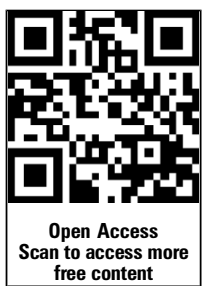

\section{SLinked}

- http://dx.doi.org/10.1136/ fetalneonatal-2012-302546

\footnotetext{
To cite: Arya VB, Flanagan SE, Kumaran A, et al. Arch Dis Child Fetal Neonatal Ed 2013;98: F356-F358.
}

\section{ABSTRACT}

Objective To characterise the phenotype and genotype of neonates born small-for-gestational age (SGA; birth weight $<10$ th centile) who developed hyperinsulinaemic hypoglycaemia $(\mathrm{HH})$.

Methods Clinical information was prospectively collected on 27 SGA neonates with $\mathrm{HH}$, followed by sequencing of $K C N J 11$ and $A B C C 8$.

Results There was no correlation between the maximum glucose requirement and serum insulin levels. Serum insulin level was undetectable in five infants (19\%) during hypoglycaemia. Six infants (22\%) required diazoxide treatment $>6$ months. Normoglycaemia on diazoxide $<5 \mathrm{mg} /$ $\mathrm{kg} /$ day was a safe predictor of resolved $\mathrm{HH}$. Sequencing of KCNJ11/ABCC8 did not identify any mutations.

Conclusions Serum insulin levels during hypoglycaemia taken in isolation can miss the diagnosis of HH. SGA infants may continue to have hypofattyacidaemic hypoketotic HH beyond the first few weeks of life. Recognition and treatment of this group of patients are important and may have important implications for neurodevelopmental outcome of these patients.

\section{INTRODUCTION}

Hyperinsulinaemic hypoglycaemia $(\mathrm{HH})$ is characterised by the unregulated secretion of insulin from pancreatic $\beta$-cells in relation to the blood glucose concentration. It is the commonest cause of severe and persistent hypoglycaemia in the neonatal and infancy periods. ${ }^{1}$ The genetic basis of congenital forms of $\mathrm{HH}$ involves defects in key genes (commonest-ABCC8 and KCNJ11 encoding the two subunits SUR1 and Kir6.2, respectively), which regulate insulin secretion from pancreatic $\beta$-cells. ${ }^{1}$ $\mathrm{HH}$ may be secondary to certain risk factors (such as maternal diabetes mellitus, perinatal asphyxia and small-for-gestational age (SGA)). In 1984, Collins and Leonard first described transient $\mathrm{HH}$ in SGA infants. ${ }^{2}$ It has not previously been documented whether the $\mathrm{HH}$ observed in infants born SGA is due to defects in the $A B C C 8 / K C N J 11$ genes. The phenotype of a large cohort of SGA neonates who developed $\mathrm{HH}$ has not been studied previously. The purpose of the present study was to report on the phenotype, clinical course and results of the sequencing of $A B C C 8 / K C N J 11$ in a cohort of infants born SGA who developed $\mathrm{HH}$.

\section{PATIENTS AND METHODS}

We prospectively studied the clinical and biochemical features of 27 SGA (defined as birth weight

\section{What is already known on this topic}

Infants born small-for-gestational age are at an increased risk to develop transient hyperinsulinaemic hypoglycaemia $(\mathrm{HH})$.

- Mutations in ABCC8/KCNJ11 genes are the commonest cause of congenital $\mathrm{HH}$.

\section{What this study adds}

- This largest series of small-for-gestational age infants who developed hyperinsulinaemic hypoglycaemia $(\mathrm{HH})$ highlights that hypofattyacidaemic hypoketotic $\mathrm{HH}$ may persist beyond the first few weeks of life.

- $\mathrm{HH}$ in these infants is diazoxide responsive and not identified to be due to mutations in $A B C C 8 / K C N J 11$ genes in this series.

- Withdrawal of diazoxide treatment when glycaemic control is maintained at a dose of $<5 \mathrm{mg} / \mathrm{kg} /$ day can be safely recommended.

$<10$ th centile) neonates with $\mathrm{HH}$ who were admitted to two tertiary paediatric referral centres (Great Ormond Street Hospital and Bristol Royal Hospital for Children). Neonates with history of perinatal asphyxia, Rhesus isoimmunisation and syndromic forms such as Beckwith-Wiedemann syndrome were excluded from the study. All were investigated at the time of clinical and biochemical hypoglycaemia to establish the diagnosis of $\mathrm{HH}$ and were commenced on diazoxide $(5-20 \mathrm{mg} / \mathrm{kg} /$ day). Diazoxide responsiveness was defined as ability to come off intravenous glucose and maintain normoglycaemia during a physiological fast. Following discharge, they were followed up at 3 -monthly intervals in outpatient clinic, where glycaemic control and diazoxide dose were reviewed. These infants were readmitted for a 24-h glucose profile and a controlled fast after stopping diazoxide for 3 days, if home glucose monitoring indicated no recurrence of hypoglycaemia on $<5 \mathrm{mg} / \mathrm{kg} /$ day of diazoxide. ${ }^{1}$ If home glucose monitoring revealed hypoglycaemic episodes, the dose of diazoxide was adjusted and the trial off diazoxide was postponed until the dose was $<5 \mathrm{mg} / \mathrm{kg} /$ day. 
Table 1 Clinical characteristics of 27 small-for-gestational age infants with hyperinsulinaemic hypoglycaemia

\begin{tabular}{ll}
\hline Age at presentation (days) & \\
$\quad$ Median (range) & $1(1-14)$ \\
Gestational age (weeks) & $37(32-42)$ \\
$\quad$ Median (range) & \\
Birth weight (g) & $2300(1100-3100)$ \\
$\quad$ Median (range) & 66.6 \\
Male (\%) & 17 \\
Ethnic origin (n) & 6 \\
Caucasian & 2 \\
Afro-Caribbean & 2 \\
Asian & 100 \\
Mixed & \\
Diazoxide responsive (\%) & $15.6(8.0-20.8)$ \\
Glucose infusion rate (mg/kg/min) & \\
Median (range) &
\end{tabular}

\section{Genetics}

Genomic DNA was extracted from peripheral leukocytes using standard procedures and the coding regions and intron/exon boundaries of the ABCC 8 and KCNJ11 genes were amplified by PCR (primers available on request). Amplicons were subsequently sequenced using the Big Dye Terminator Cycler Sequencing Kit V.3.1 (Applied Biosystems, Warrington, UK) according to manufacturer's instruction and reactions were analysed on an ABI 3730 Capillary sequencer (Applied Biosystems, Warrington, UK). Sequences were compared with the reference sequences (NM 000525.3 and NM 000352.3) using Mutation Surveyor v3.24 software (SoftGenetics, State College, Pennsylvania, USA).

\section{RESULTS}

Clinical characteristics and course

The phenotypic details of 27 SGA infants with $\mathrm{HH}$ are summarised in table 1.

The plasma insulin levels were inappropriately elevated during hypoglycaemia in 22 infants $(81 \%)$. The remaining five infants (19\%) had inappropriately low $\beta$-hydroxybutyrate and nonesterified fatty acids during hypoglycaemia as supportive biochemical evidence of $\mathrm{HH}$. All infants required glucose infusion rate of $\geq 8 \mathrm{mg} / \mathrm{kg} / \mathrm{min}$ to maintain normoglycaemia (median $15.6 \mathrm{mg} / \mathrm{kg}$ / min). There was no correlation found between maximum glucose infusion rate to maintain normoglycaemia and plasma insulin level during hypoglycaemia (figure 1). Most (89\%) responded to mildmoderate doses of diazoxide $(5-10 \mathrm{mg} / \mathrm{kg} /$ day $)$, with three infants (11\%) needing high doses $(15-20 \mathrm{mg} / \mathrm{kg} /$ day $)$.

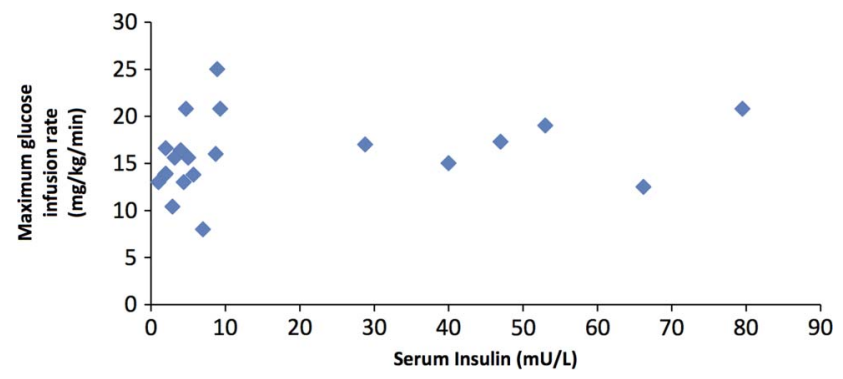

Figure 1 Figure demonstrating the lack of correlation between maximum glucose infusion rate and plasma insulin concentrations.
Glucose profile and fasting responses at resolution of hyperinsulinism

In all, 17 infants (63\%) underwent a 24 h glucose profile and controlled fast after stopping diazoxide to demonstrate resolution of hyperinsulinism. None of these infants recorded hypoglycaemia during a 24-h glucose profile. All infants maintained blood glucose concentrations above $3.0 \mathrm{mmol} / \mathrm{l}$ at the end of fast appropriate for their age, with 13 infants maintaining blood glucose concentrations $>3.5 \mathrm{mmol} / \mathrm{l}$. All infants showed appropriate plasma insulin suppression and elevation of plasma $\beta$-hydroxybutyrate/nonesterified fatty acids as evidence of resolution of $\mathrm{HH}$.

\section{Genetic results}

Sequencing did not reveal mutations in $A B C C 8 / K C N J 11$ genes in 25/27 DNA samples analysed.

\section{DISCUSSION}

This is the largest case series of SGA neonates with $\mathrm{HH}$ that reports the clinical characteristics and $A B C C 8 / K C N J 11$ sequencing results in a cohort of 27 subjects. Collins et al first described transient $\mathrm{HH}$ in three SGA infants and subsequently reported $\mathrm{HH}$ in five of 27 SGA neonates studied, suggesting it is common in SGA neonates. ${ }^{2}{ }^{3}$ It is important to recognise this as neonates with $\mathrm{HH}$ are especially prone to the complications of hypoglycaemia because of their inability to generate alternative fuels, such as ketone bodies.

In our study, despite other evidence of hyperinsulinism, plasma insulin levels were undetectable during hypoglycaemia in 5/27 SGA neonates. We did not find any correlation between the maximum glucose infusion rate required to maintain normoglycaemia and plasma insulin levels. This is explained by the short half-life of insulin of 4-5 min and rapid clearance by the liver before it reaches peripheral circulation. In a study by Hoe et $a l,{ }^{4}$ plasma insulin was inappropriately elevated in only 11 of 24 neonates who had transient prolonged neonatal hyperinsulinism. Our results corroborate these findings and highlight the value of other parameters (apart from plasma insulin) in the diagnosis of $\mathrm{HH}$.

In this case study of SGA infants referred to two tertiary hospitals with $\mathrm{HH}$, all infants responded to diazoxide, though the dose was variable ranging from 5 to $20 \mathrm{mg} / \mathrm{kg} /$ day. Diazoxide was successfully stopped in $21(78 \%)$ patients by 6 months of age. In the remaining six infants, five needed diazoxide until 10 months of age and one infant needed prolonged treatment until 22 months. In a study of 26 neonates with hyperinsulinism which included 7 SGA neonates, $\mathrm{HH}$ resolved by a median age of 181 days (range, $18-403$ days). ${ }^{4}$ Fafoula et al ${ }^{5}$ also reported prolonged transient $\mathrm{HH}$ up to 9 months in SGA infants.

In our cohort, no recurrence of $\mathrm{HH}$ was noticed after stopping diazoxide. This highlights that with the management protocol whereby diazoxide was stopped when good glycaemic control was maintained with diazoxide dose $<5 \mathrm{mg} / \mathrm{kg} /$ day, the risk of recurrence of hypoketotic hypoglycaemia brain injury to these vulnerable infants is avoided.

To our knowledge, no previous study has reported on the genetic results of SGA neonates who developed $\mathrm{HH}$. In our study, no mutations in $A B C C 8 / K C N J 11$ were identified in these SGA infants. However, rarer genetic causes of $\mathrm{HH}$ due to mutations in GLUD1, HADH, GCK and HNF4A were not excluded.

\section{CONCLUSIONS}

SGA infants may continue to have hypofattyacidaemic hypoketotic $\mathrm{HH}$ beyond the first few weeks of life. Recognition and 
treatment of this group of patients are important and may have important implications for neurodevelopmental outcome of these patients. Plasma insulin levels during hypoglycaemia taken in isolation can miss the diagnosis of $\mathrm{HH}$ and hence other parameters (such as glucose infusion rates and production of free fatty acids/ketone bodies during hypoglycaemia) must be considered in the diagnosis. Withdrawal of diazoxide treatment when glycaemic control is maintained at a dose of $<5 \mathrm{mg} / \mathrm{kg} /$ day can be safely recommended.

Finally, the genetic aetiology of HH in SGA infants is not understood and, in this study, mutations in the common genes implicated in the aetiology of congenital hyperinsulinism were not identified. Further studies are required to understand the underlying mechanism of $\mathrm{HH}$ in these infants.

Acknowledgements This study was funded by the Wellcome Trust (081188/A/06/ Z). VBA was funded by MRC, SEF was the Sir Graham Wilkins Peninsula Medical School Research Fellow, SE was funded by the Royal Devon \& Exeter NHS Foundation Trust Research \& Development Directorate and RRK was funded by NIHR.

Contributors VBA collected and analysed the data and wrote the manuscript. SEF performed the genetic studies and reviewed the manuscript. AK and JPS collected the data and critically reviewed the manuscript. SE and KH conceptualised the study and critically reviewed the manuscript. RRK conceptualised the study, analysed the data and critically reviewed the manuscript. All authors contributed to the approval of the final version of the manuscript. RRK is the guarantor of this work.
Funding This work was funded by the Wellcome Trust (081188/A/06/Z). Competing interests None.

Ethics approval Ethics Committee of Great Ormond Street Hospital for Children and the Institute of Child Health, University College London.

Provenance and peer review Not commissioned; externally peer reviewed.

Open Access This is an Open Access article distributed in accordance with the Creative Commons Attribution Non Commercial (CC BY-NC 3.0) license, which permits others to distribute, remix, adapt, build upon this work non-commercially, and license their derivative works on different terms, provided the original work is properly cited and the use is non-commercial. See: http://creativecommons.org/ licenses/by-nc/3.0/

\section{REFERENCES}

1 Kapoor RR, Flanagan SE, James C, et al. Hyperinsulinaemic hypoglycaemia. Arch Dis Child 2009;94:450-7.

2 Collins JE, Leonard JV. Hyperinsulinism in asphyxiated and small-for-dates infants with hypoglycaemia. Lancet 1984;2:311-13.

3 Collins JE, Leonard JV, Teale D, et al. Hyperinsulinaemic hypoglycaemia in small for dates babies. Arch Dis Child 1990;65:1118-20.

4 Hoe FM, Thornton PS, Wanner LA, et al. Clinical features and insulin regulation in infants with a syndrome of prolonged neonatal hyperinsulinism. J Pediatr 2006:148:207-12.

5 Fafoula $\mathrm{O}$, Alkhayyat $\mathrm{H}$, Hussain K. Prolonged hyperinsulinaemic hypoglycaemia in newborns with intrauterine growth retardation. Arch Dis Child Fetal Neonatal Ed 2006;91:F467. 